\title{
REVISIÓN DE LOS TRABAJOS ORIGINALES QUE ANALIZAN LOS EFECTOS DE LA CONTAMINACIÓN ATMOSFÉRICA EN LA MORTALIDAD, 1994-1998
}

\author{
José M.a Tenías Burillo (1,2), Ferran Ballester Díez (1), Sylvia Medina (3) y Antonio Daponte \\ Codina (4) \\ (1) Unidad de Epidemiología y Estadística. Institut Valencià d'Estudis en Salut Pública-IVESP. Dirección General \\ de Salud Pública. Generalitat Valenciana. \\ (2) Servicio de Medicina Preventiva. Hospital Lluís Alcanyís. Xàtiva, Valencia. \\ (3) Reseau National de Santé Publique. Saint Maurice. Francia. \\ (4) Escuela Andaluza de Salud Pública. Granada.
}

\section{RESUMEN}

Se revisan los diferentes estudios publicados e indexados en Medline y el Índice Médico Español (IME) desde el año 1984 hasta el año 1998 (IME: desde 1971 a 1996).

En una primera fase se evaluó la producción cuantitativa de publicaciones que versan sobre los efectos de la contaminación en la salud, así como la proporción de éstas que analizan la mortalidad como indicador de salud. En una segunda fase se revisó de forma pormenorizada los trabajos originales publicados en el periodo de enero de 1994 a junio de 1998.

La mayoría de los artículos originales son estudios agregados de series temporales que analizan, con el día como unidad de análisis, la relación entre los niveles de contaminación y la mortalidad por causas no accidentales.

También sc resumen otros artículos originales con un diseño diferente al de series temporales, destacando diferentes estudios de cohortes que relacionan los niveles de contaminación con la mortalidad a nivel individual y que confirman los hallazgos de estudios ecológicos previos.

Los contaminantes analizados con mayor frecuencia son las particulas en sus diferentes tipos, aunque tambićn son comuncs los estudios sobre el efecto del dióxido de azufre, dióxido de nitrógeno, ozono y monóxido de carbono.

La metodología de análisis más utilizada es la regresión de Poisson con la incorporación de diferentes variables de confusión como estacionalidad y tendencia, temperatura y humedad y efectos de calendario, fundamentalmente.

En los estudios revisados predominan los hallazgos positivos y significativos, sicndo consistentes sobre todo para particulas, que es el contaminante más analizado. Los trabajos que estudian otros contaminantes, aunque menos numerosos, también indican una relación significativa entre sus niveles y la mortalidad.

Palabras clave: Contaminación atmosférica. Efectos adversos. Mortalidad. Revisión.

Correspondencia:

Ferran Ballester Díez.

Unidad de Epidemiología y Estadistica. Institut Valencià d'Estudis en Salut Pública-IVESP.

Joan de Garay, 21

46017 Valencia.

Teléfono 96-3869369

Fax 96-3869370

Correo electrónico: fballest@san.gva.es

\section{ABSTRACT \\ Review of Original Papers Analyzing the Impact of Air Pollution on the Mortality, 1994-1998}

The different studies published and indexed in Medline and in the Spanish Medical Index (IME) from 1984 up to 1998 (IME: 1971-1996) are reviewed.

To start with, an assessment was made of the number of publications put out which have to do with the impact of pollution on human health as well as the percentage thereof which analyze the mortality as a health indicator. Afterward, the original works published within the January 1994-June 1998 period were reviewed in detail.

Most of the original articles are combined time series studies which analyze, on a single-day basis, the relationship between the levels of pollution and the mortality for non-accidental causes.

Other original articles were also summarized based on an approach other than the time series one, highlighting different cohort studies which relate the levels of pollution to the mortality on an individual level and which confirm the findings of prior ecological studies.

The pollutants analyzed most often are the different types of particles, although studies are also common regarding the impact of sulfur dioxide, nitrogen dioxide, ozone and carbon monoxide.

The method of analysis most used is the Poisson regression, into which different confusion variables bearing an impact thereon such as seasonality and tendency, temperature and relative humidity and day of the year are basically added.

In the studies reviewed, the positive significant findings prevail, being consistent especially for particles, which is the pollutant most analyzed. The articles studying other pollutants, although fewer in number, also indicate a significant relationship between pollution levels and the mortality.

Key words: Air pollution. Advcrse impact. Mortality. Review. 


\section{INTRODUCCIÓN}

El interés científico y social acerca de las consecuencias de la contaminación atmosférica sobre la salud de los ciudadanos se ha incrementado de forma notable en la última década ${ }^{1}$. En fechas recientes, han visto la luz varios trabajos de meta-análisis que resumen la asociación que, a corto plazo, se produce entre contaminación atmosférica y mortalidad en diversas ciudades europeas ${ }^{2-4}$ y americanas $^{5,6}$. Estos estudios aportan evidencias firmes que apoyan la consistencia de los resultados sobre la relación entre contaminación y salud. Además, durante este periodo se han producido un número importante de investigaciones que, siguiendo en algunos casos metodologías más o menos estandarizadas y en otros con diseños menos frecuentes, aportan nuevas evidencias, confirmando hallazgos previos y, en ocasiones, abriendo futuras líneas de investigación. Entre éstos destacan los estudios de cohortes realizados en los Estados Unidos de América (EEUU) en los que se ha encontrado asociación entre los niveles de contaminación y mortalidad a nivel individual ${ }^{7,8}$. Por otro lado, en los últimos años, se han llevado a cabo trabajos en los que se analiza el efecto de la contaminación sobre indicadores de mortalidad menos habituales, como es la muerte súbita infantil ${ }^{9,10}$ o la mortalidad intrauterina ${ }^{\mathrm{ll}}$.

Por todo ello, creemos importante incluir en este número de la Revista Española de salud Pública una revisión, lo más exhaustiva posible, de la literatura publicada sobre el tema en los últimos cinco años. En relación a lo publicado en periodos anteriores, el lector puede acudir a una revisión anterior editada en esta misma revista ${ }^{12}$.

El objetivo de esta revisión es describir la evolución cuantitativa de los estudios sobre los efectos de la contaminación atmosférica en la salud desde 1971 en el ámbito nacional y desde el año 1984 en el ámbito internacional, así como examinar los resúmenes de todos los trabajos publicados sobre el impacto de la contaminación atmosférica en la mortalidad en el periodo comprendido entre 1994 y 30 de junio de 1998 y presentar un revisión de los trabajos originales identificados.

\section{MATERIAL Y MÉTODOS}

\section{Criterios seguidos en la búsqueda}

La información sobre el matcrial bibliográfico se ha obtenido a partir de dos bases de datos documentales, en su versión para CD-Rom: Medline (producida por la National Library of Medicine, Bethesda, EEUU) e Índice Médico Español, IME, (producida por el Centro de Documentación e Informática del Consejo Superior de Investigaciones Científicas).

Para la consulta en Medline se utilizaron los términos del Thesaurus «air pollution»y «air pollutants», ambos con el subdescriptor «adverse effects», limitando la búsqueda al campo del término genérico «human» (estrategia 1). Además se realizó otra búsqueda añadiendo «mortality» como término del Medical Subject Headings (MeSH) (estrategia 2). Para el período que abarca desde el año 1984 a 1997 se obtuvo el número anual de publicaciones totales y de publicaciones recuperadas con ambas estrategias. Para el periodo de 1 de enero de 1994 a 30 de junio de 1998 se recuperó toda la información disponible en Medline siguiendo la estrategia 2. Para completar exhaustivamente la recuperación de artículos, se realizó otra búsqueda combinando las palabras clave «air pollution» y «mortality» como lenguaje libre (estrategia 3). Se confeccionó un listado conjunto de las referencias recuperadas con las estrategias 2 y 3 ( 240 y 195 respectivamente). De la combinación de las dos búsquedas se confeccionó un listado con todos los campos que aparecen en Medline de un total de 316 referencias no comunes a las que se añadió una más de un artículo publicado en junio de $1998^{11}$ y que aún no estaba actualizada en el momento de la búsqueda en la versión para $C D$-Rom utilizada. 


\section{Selección de los artículos}

Después de revisar los títulos y resúmenes de los 317 manuscritos recuperados, se seleccionaron para un posterior análisis 145 trabajos. Los criterios de selección fueron:

- que el manuscrito tratara de contaminación atmosférica exterior («outdoor») en inglés).

- que el contaminante fuera de naturaleza química.

- que se relacionara la contaminación con algún indicador de mortalidad ocurrida por causas no accidentales.

- que fuera un estudio realizado en humanos.

Tras la aplicación de estos criterios se descartaron 172 documentos, 131 de ellos por no versar sobre contaminación atmosférica exterior ( 25 de contaminación interior - «indoor»-, 68 de contaminantes ocupacionales diferentes a los componentes de la contaminación ambiental exterior, y 49 sobre el nivel ambiental del humo de tabaco), en 9 no se relacionó la contaminación con ningún indicador de mortalidad, en 6 el estudio se realizó en animales de laboratorio, y en 8 casos se relacionó con un indicador de mortalidad por accidentes (en su mayoría suicidios). En 18 documentos no se identificó el tipo de contaminación estudiada.

Los 145 documentos seleccionados se clasificaron según el tipo de manuscrito en originales $(69 ; 47,6 \%)$, meta-análisis $(4 ; 2,8 \%)$, artículos de revisión $(53 ; 36,6 \%)$, cartas o artículos de opinión $(14 ; 9,7 \%)$; en 5 casos $(3,4 \%)$ no se pudo clasificar. Según la zona geográfica en la que se llevó a cabo el estudio (en el caso de los originales y meta-análisis) o la procedencia del primer autor para el resto de manuscritos, la mayoría de los documentos seleccionados proceden de América $(63 ; 43,4 \%, 49$ de Estados Unidos) y Europa $(66 ; 45,5 \%)$. El idioma en el que se redactaron los trabajos fue el inglés en la mayoría de los casos $(120 ; 82,8 \%)$ y el resto en francés, alemán, italiano, castellano, ruso, polaco checo, danés, chino y húngaro.

Las principales características de los 73 trabajos originales (incluyendo los 4 meta-análisis) se resumen en la tabla 1.

Tabla 1

Características de los artículos originales recuperados sobre contaminación atmosférica y mortalidad

\begin{tabular}{|c|c|c|}
\hline & & $n(\%)$ \\
\hline \multirow[t]{7}{*}{ Diseño del estudio } & Series temporales & $42(57,5 \%)$ \\
\hline & Geográficos & $12(16,4 \%)$ \\
\hline & Cohortes & $4(5,5 \%)$ \\
\hline & Casos-control & $1(1,4 \%)$ \\
\hline & Transversales & $1(1,4 \%)$ \\
\hline & Estudios sobre metodología de análisis & $7(9,6 \%)$ \\
\hline & Estudios de laboratorio en humanos & $6(8,2 \%)$ \\
\hline \multirow[t]{6}{*}{ Zona de procedencia del estudio } & América & \\
\hline & EEUU & $19(27,1 \%)$ \\
\hline & Otros paises & $9(11,3 \%)$ \\
\hline & Europa & $37(50,7 \%)$ \\
\hline & Asia & $6(8,2 \%)$ \\
\hline & Oceanía & $2(2,7 \%)$ \\
\hline \multirow[t]{2}{*}{ Idioma } & Inglés & $68(93,2 \%)$ \\
\hline & Otros idiomas & $5(6,8 \%)$ \\
\hline
\end{tabular}


Para la búsqueda correspondiente a la base de datos IME se utilizó la palabra clave «contaminación» en combinación con «aire» o «atmosfer*» para el periodo 1971 a 1996. Las búsquedas bibliográficas se realizaron durante el mes de agosto de 1998.

Con el objetivo de valorar el impacto de la contaminación atmosférica sobre la mortalidad por causas distintas a las externas, se escogieron 60 documentos, descartando los estudios de laboratorio y metodológicos. Tampoco se seleccionaron para su revisión aquellos manuscritos escritos en otra lengua que no fuera el inglés, castellano o francés. De los 55 documentos finales, ocho no pudieron recuperarse por problemas de acceso a la fuente original.

Se repartieron los 47 trabajos originales seleccionados entre los cuatro revisores. Cada revisor leyó los artículos asignados y completó una ficha que recogía la información más relevante.

\section{RESULTADOS}

\section{Referencias del periodo 1984-1997}

La tabla 2 muestra, para cada uno de los años tenidos en cuenta, el número total de trabajos referenciados en la base de datos Medline, el número de artículos recuperados con la estrategia de efectos adversos de la contaminación atmosférica en humanos y los recuperados con la estrategia que estudia específicamente su impacto en la mortalidad. El número de referencias recuperado por ambas estrategias se ha incrementado en la segunda parte del periodo estudiado, siendo algo menor este aumento en términos relativos. El menor número de referencias para el año 1997 se debe a que este año no estaba completamente actualizado en las fechas en que se llevó a cabo la revisión.

La figura 1 muestra la evolución anual del número de artículos recuperados de la base de datos IME, desde 1971 hasta 1996. El pico que se observa en el año 1984 coincide con la edición de un número monográ-

Tabla 2

Número de referencias recuperadas en Medline por año según estrategia de búsqueda

\begin{tabular}{|lccccc|}
\hline Año & Total referencias & Estrategia I & \% sobre referencias & Estrategia 2 & \% sobre Estrategia 1 \\
\hline 1984 & 297.292 & 363 & 0,12 & 17 & 4,68 \\
1985 & 307.572 & 374 & 0,12 & 14 & 3,74 \\
1986 & 320.510 & 401 & 0,13 & 14 & 3,49 \\
1987 & 337.197 & 446 & 0,13 & 23 & 5,16 \\
1988 & 354.215 & 439 & 0,12 & 23 & 5,24 \\
1989 & 369.510 & 585 & 0,16 & 39 & 6,67 \\
1990 & 376.690 & 613 & 0,16 & 36 & 5,87 \\
1991 & 375.811 & 662 & 0,18 & 42 & 6,34 \\
1992 & 377.343 & 584 & 0,15 & 36 & 6,16 \\
1993 & 382.420 & 573 & 0,15 & 42 & 7,33 \\
1994 & 388.331 & 616 & 0,16 & 40 & 6,49 \\
1995 & 394.785 & 589 & 0,15 & 48 & 8,15 \\
1996 & 395.876 & 724 & 0,18 & 58 & 8,01 \\
1997 & 280.351 & 437 & 0,16 & 27 & 6,18 \\
\hline TOTAL & 4.957 .903 & 7.406 & 0,15 & 459 & 6,20 \\
\hline
\end{tabular}

Estrategia 1: «air pollution» o «air pollutants» como términos del Thesaurus, anubos con el subdescriptor «adverse effects» descriptores y «human» como término genérico.

Estrategia 2: estrategia 1 añadiendo «mortality» como término del Medical Subject Heading. 
Figura 1

Número de referencias recuperadas de la base bibiliográfica IME (Índice Médico Español) utilizando la palabra clave «contaminación» en combinación con «aire» n «atmosfer*" según año de publicación. 1971-1996

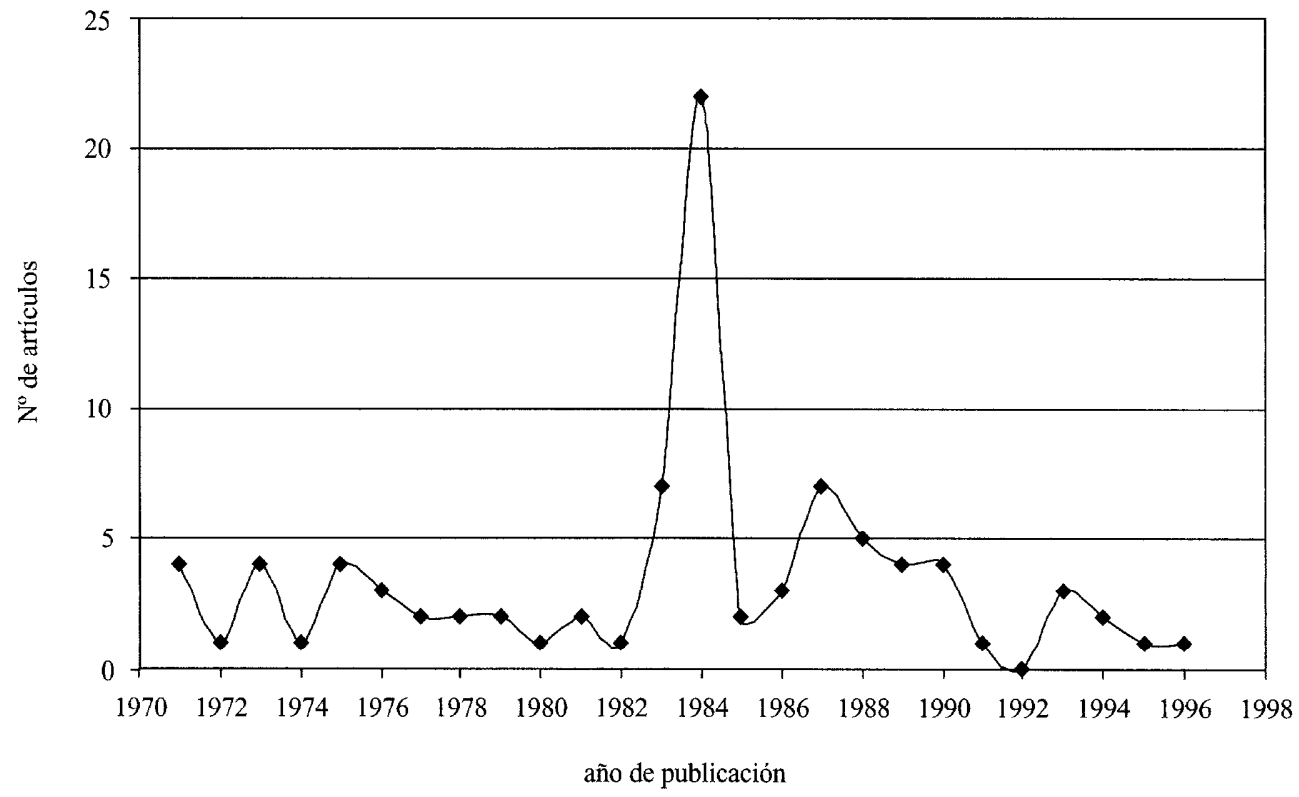

fico de la Gaseta Sanitaria de Barcelona ${ }^{13}$. La tabla 3 muestra las revistas en las que se han publicado los trabajos recuperados en la base del IME. En ella destacan, por el número de artículos publicados, la Revista de Sanidad e Higiene Pública y la Gaseta Sanitària de Barcelona. Quince de los 19 artículos de ésta última corresponden al suplemento anteriormente mencionado. Ambas revistas han cambiado de nombre. La Revista de Sanidad e Higiene Pública pasó a denominarse Revista Española de Salud Pública a partir de 1995. La Gaseta Sanitària de Barcelona desapareció como tal y dejo paso a Gaceta Sanitaria en 1987 (órgano de difusión de la Sociedad Española de Salud Pública y Administración Sanitaria). Desde entonces y hasta 1996 únicamente se había publicado un trabajo sobre la contaminación atmosférica. Recientemente han aparecido en Gaceta Sanitaria dos artículos, uno analizando la relación de la conta- minación atmosférica sobre la mortalidad en la ciudad de Madrid ${ }^{14}$ y el otro evaluando su impacto sobre la salud de los residentes en Barcelona ${ }^{15}$.

Además de las dos anteriores, las revistas médicas españolas que han publicado más artículos sobre contaminación atmosférica pertenecen al campo de la pediatría y al de la salud laboral, con 10 artículos en cada uno de estas especialidades. También destacan otras dos revistas: Medicina Clínica y Gaceta Médica de Bilbao, con 6 publicaciones recuperadas en cada una. Por último, revistas de las especialidades de alergia, inmunología, microbiología, toxicología y medicina legal, entre otras, completan el conjunto de publicaciones periódicas médicas españolas, en las que se han difundido los resultados de las investigaciones sobre los peligros para la salud derivados de la contaminación del aire. 
Tabla 3

Revistas y número de artículos de los trabajos sobre contaminación atmosférica recuperados de la base de datos del IME. 1971-1996

\begin{tabular}{|lc|}
\hline \multicolumn{1}{|c|}{ Revistas } & $\begin{array}{c}\text { Número } \\
\text { de artículos }\end{array}$ \\
\hline Allergologia et Immunopathologia & 2 \\
Anales Españoles de Pediatría & 9 \\
Annals de Medicina & 2 \\
Archivos de Medicina del deporte & 1 \\
Archivos de Pediatría & 1 \\
Centro de Salud & 1 \\
Gaceta Médica de Bilbao & 6 \\
Gaceta Sanitaria & 1 \\
Gaseta Sanitària de Barcelona & 19 \\
Medicina Clínica & 6 \\
Medicina Intensiva & 1 \\
Medicina y Seguridad del Trabajo & 3 \\
Microbiología & 3 \\
Revista de Sanidad e Higiene Pública & 23 \\
Revista de Toxicología & 1 \\
Revista Española de Alergología e Inmu- & \\
nología Clínica & 1 \\
Revista Española de Medicina Legal & 1 \\
Revista Española de Salud Pública & 1 \\
Salud y Trabajo & 7 \\
\hline TOTAL & 89 \\
\hline
\end{tabular}

\section{Artículos originales del periodo enero 1994-junio 1998}

Entre las referencias recuperadas de la base de datos IME, no se ha encontrado ninguna correspondiente a los años 1994 a 1996 que cumpla los criterios expuestos en la sección de método.

Los 47 artículos originales recuperados a partir de la búsqueda en Medline correspondiente al periodo enero de 1994 a junio de 1998 se dividieron en dos grandes grupos. En el primero de ellos se incluyeron aquellos trabajos en los que se había utilizado la metodología de series temporales y en los que la unidad de análisis era el día. Este fue el grupo más numeroso (38 artículos). Entre los trabajos no englobados en el grupo anterior se encuentran un total de nueve trabajos que han utilizado diseños de análisis geo- gráfico, estudios de cohortes, estudios de episodios o estudios mixtos. Se confeccionan dos tablas en las que se resumen las principales características y resultados de estos estudios y que, por motivos de espacio, no se presentan en este artículo (pueden obtenerse solicitándolas a los autores).

\section{Estudios de series temporales}

Los estudios de series temporales son, con mucho, el diseño más frecuentemente utilizado en los últimos años en el análisis de la relación a corto plazo entre contaminación atmosférica y mortalidad. Del total de 38 artículos seleccionados, 34 son artículos originales y los otros cuatro restantes son meta-análisis que combinan los resultados obtenidos en distintos grupos de ciudades ${ }^{2,3,5.6}$. En la mayoría de los originales se evaluó lo que ocurre en ciudades de Europa (17 casos) o de los Estados Unidos (8 trabajos, de los cuales tres corresponden a Filadelfia $\left.{ }^{16-18}\right)$. Nueve de los artículos originales sobre ciudades europeas pertenecen al proyecto APHEA ${ }^{19-27}$. Otros seis trabajos se han realizado en ciudades de Latinoamérica, 2 en China y uno en Brisbane (Australia).

\section{Población, periodo y causas de muerte a estudio}

La población a estudio suele ser la de las personas residentes en las ciudades y se excluyen, por lo general, las defunciones ocurridas fuera de la ciudad de residencia. Las poblaciones más numerosas corresponden a las megalópolis latinoamericanas (México, Sao Paulo), la de Beijing, en China, o la de Londres. También se ha estudiado el impacto de la contaminación sobre la mortalidad en el conjunto de algunos países de dimensiones reducidas como Bélgica o Taiwan. Las ciudades con menor población entre las estudiadas son Lyon (Francia) y los condados del Valle de Utah, en EEUU. 
Las series más antiguas son las de los estudios de Filadelfia; dos de ellas comienzan en el año $1973^{16,17}$ y la otra en $1974^{18}$. El periodo más reciente entre los revisados corresponde al verano de 1994 en Bélgica ${ }^{28,29}$, coincidiendo con una de ola de calor que ocurrió ese año en Europa. Sin embargo, en la mayor parte de los casos los periodos de estudio oscilan entre mediados de los años ochenta y comienzos de los noventa.

En 30 de los 34 originales revisados se incluye como variable dependiente el número de defunciones por todas las causas (por lo general con exclusión de las muertes por causas externas). Únicamente en cuatro de los trabajos no se ha estudiado dicho grupo. En tres de ellos se ha analizado exclusivamente la mortalidad por causas respiratorias $^{19,23,30}$ y en el otro Knöbel et al ${ }^{10}$ examinan la posible relación de la contaminación con la muerte súbita infantil (Clasificación Internacional de Enfermedades, 9. ${ }^{a}$ revisión, CIE 9, 798 ${ }^{31}$ ). Además de las defunciones por todas las causas, los grupos específicos más estudiados son las defunciones por causas respiratorias ( 24 de los 30 manuscritos que estudian todas las causas) y por causas del aparato circulatorio (en 22 originales). Entre las primeras, además del grupo de todas las respiratorias (CIE 9 460-519, caso del proyecto APHEA), bastantes autores examinan causas más específicas de muerte, entre ellas las defunciones por neumonía (CIE 9, 480-487) y la enfermedad pulmonar obstructiva crónica (CIE 9, 490 a 496, excepto 493 que se reserva para el asma - la clasificación de este grupo es variable según autores). Algo similar ocurre con las causas del aparato circulatorio, o bien se estudia un grupo amplio de enfermedades (lo más habitual CIE 9 390-459, como en el proyecto APHEA, o algo más específico 390-448). En los trabajos del proyecto APHEA se incluyen también las defunciones por causas digestivas (CIE 9 520-579) como grupo de control. Otros autores ${ }^{32-34}$ han incluido las defunciones por cáncer (CIE 9 140-208) entre las causas estudiadas 0 , más específica- mente las defunciones por cáncer de put món ${ }^{16}$ (CIE 9 162).

En la mayor parte de los 30 originales que analizan el total de defunciones se incluyen todos los grupos de edad. Sólo cinco trabajos estudian exclusivamente otros grupos de edad. Entre ellos, Saldiva et al, en Sao Paulo realizan un estudio en el grupo de menores de 5 años ${ }^{30}$, y otro en las personas mayores de 65 años ${ }^{35}$. En la misma ciudad, Pereira et $a^{11}$ evalúan el impacto de la polución aérea en la mortalidad intrauterina (fetos de más de 28 semanas). En los estudios en Bélgica sobre el impacto de la contaminación atmosférica durante una ola de calor se analizan por separado las defunciones de los menores y mayores de 65 años $^{28}$, o se examina únicamente el grupo de los mayores de 65 años ${ }^{29}$.

En cuanto al género, prácticamente ningún estudio examina las posibles diferencias entre hombres y mujeres, excepto Ostro et al en Santiago de Chile ${ }^{36}$ e Ito y Thurston en el Condado de Cook $^{34}$.

\section{Contaminantes}

Los contaminantes más estudiados son las partículas en suspensión y el dióxido de azufre $\left(\mathrm{SO}_{2}\right)$. Las primeras incluyen diferentes indicadores, como las partículas con un diámetro aerodinámico de mediana menor o igual a $10 \mu \mathrm{m}\left(\mathrm{PM}_{10}\right)$ en 10 ocasiones, o los humos negros («black smoke») y el total de partículas en suspensión (TSP), en 9 casos cada indicador. Con mucha menor frecuencia se han utilizado otros indicadores de partículas como las partículas de diámetro aerodinámico de mediana menor o igual a 2,5 $\mu \mathrm{m}\left(\mathrm{PM}_{2.5}\right), 13 \mu \mathrm{m}\left(\mathrm{PM}_{13}\right), \mathrm{u}$ otros diámetros como 3,5 ó $7 \mu \mathrm{m}$. La visibilidad óptica en los acropuertos ha sido utilizada en dos trabajos ${ }^{10,37}$ como indicador de los niveles de partículas finas en el aire. $\mathrm{El} \mathrm{SO}_{2}$ es el contaminante específico que se ha estudiado con mayor frecuencia, 
en 20 de los 34 originales como la media diaria.

Los indicadores de contaminación fotoquímica también se han analizado en bastantes de los manuscritos revisados. El ozono se ha estudiado 7 veces como media diaria o como valor máximo en una hora y 5 veces como media de 8 horas. El dióxido de nitrógeno $\left(\mathrm{NO}_{2}\right), 7$ veces como media diaria y 5 como valor máximo en una hora. Junto con los anteriores el monóxido de carbono (CO) ha sido examinado en 8 casos, 5 como la media diaria y las 3 restantes como la máxima de 8 horas.

Las áreas urbanas de Latinoamérica presentan los niveles más altos de contaminación. En México Distrito Federal entre 1990 y $1992^{38}$ las TSP alcanzan $204 \mu \mathrm{g} / \mathrm{m}^{3}$, el SO $152 \mu \mathrm{g} / \mathrm{m}^{3}$, y el CO $6,6 \mathrm{mg} / \mathrm{m}^{3}$, los tres expresados como la media diaria. Los niveles de ozono se sitúan como los más elevados de los revisados con valores de $188 \mu \mathrm{g} / \mathrm{m}^{3}$ para la media de 8 horas y de $310 \mu \mathrm{g} / \mathrm{m}^{3}$ para los valores máximos de 1 hora. En Sao Paulo destacan los niveles que se alcanzan de $\mathrm{NO}_{2}, 157 \mu \mathrm{g} / \mathrm{m}^{3}$ como media diaria de los años 1991 y $1992^{11}$. Santiago de Chile es otra ciudad donde la contaminación del aire alcanza niveles elevados (media diaria de $\mathrm{PM}_{10}$ de 1989 a $1991=115 \mu \mathrm{g} / \mathrm{m}^{3}$ ). Las aglomeraciones industriales y urbanas del continente asiático también son zonas donde se presentan problemas graves de contaminación. La única ciudad asiática estudiada con este tipo de diseño es la capital de China, Beijing $^{32}$. Allí durante el año 1989 las partículas totales en suspensión registraron las concentraciones más altas de todas las revisadas, $375 \mu \mathrm{g} / \mathrm{m}^{3}$ de media diaria.

Entre las ciudades europeas estudiadas, las que presentan mayores índices de contaminación por partículas y $\mathrm{SO}_{2}$ son Milán ${ }^{23}$ y Atenas $^{26}$, junto con las ciudades de Polonia ${ }^{20}$ y de la Alemania del Este ${ }^{40}$. Las ciudades norteamericanas, por lo general, presentan niveles moderados de contaminación del aire, aunque en algunos casos se registran valores importantes de partículas como en Steubenville ${ }^{5}$ con $111 \mu \mathrm{g} / \mathrm{m}^{3}$ de media diaria o de ozono, como en el condado de $\mathrm{Cook}^{34}$, con niveles de 24 horas de $76,2 \mu \mathrm{g} / \mathrm{m}^{3}$.

\section{Metodología de análisis}

En todos los originales revisados se utilizan métodos de regresión para analizar la relación entre las variaciones en los nivcles de contaminación y el número de defunciones diarias.

La regresión de Poisson es el método elegido por la mayoría de autores. Los nueve originales que corresponden a grupos participantes en el proyecto APHEA ${ }^{19-27}$ y otros dos trabajos que explícitamente lo mencionan ${ }^{41,42}$, utilizaron una metodología estandarizada que permite la comparabilidad de resultados. Los detalles metodológicos del procedimiento se explican en otra parte ${ }^{43,44}$. Brevemente, se ajustaron modelos de regresión de Poisson autoregresiva para cada causa de muerte controlando por las principales variables de confusión, que incluyen: estacionalidad y tendencia a largo plazo, temperatura y humedad diarias, día de la semana y festivos, incidencia de gripe y otros acontecimientos inusuales, como huelgas, olas de calor o grandes fastos. La forma funcional de las variables de temperatura y humedad, o su interacción, se decidió de acuerdo con las condiciones meteorológicas locales después del examen descriptivo y analítico de los datos. Se probaron los retardos de los contaminantes hasta el tercer orden (hasta el quinto orden en el caso del ozono) y los promedios de valores de varios días consecutivos de contaminantes y se aplicaron pruebas diagnósticas para asegurar la adecuación de los modelos. Para cada ciudad se eligió el indicador de contaminación que mejor ajustaba. Se examinó también la posible modificación de efecto según se tratara de los meses más cálidos o los más fríos. Por último, sc desarrollaron modelos con más de un contaminante para valorar la 
independencia de sus efectos respecto a los otros contaminantes.

Las variables de control utilizadas en el proyecto APHEA son las que se han usado, en una u otra forma, en la mayoría de trabajos. Algunos autores han incluido otras variables meteorológicas, como la presión atmosférica, o también los pólenes ${ }^{33}$.

Además de los 11 trabajos anteriores, 14 más de $\operatorname{los} 23$ originales restantes utilizan también regresión de Poisson como método de elección. Otros tres trabajos ${ }^{11,36,37}$ utilizan la regresión de Poisson junto con el análisis de regresión múltiple, que es la scgunda aproximación más utilizada, y cinco trabajos más usan ésta última como método de elección. Por último, Salinas y Vega en el Área Metropolitana de Santiago de Chile ${ }^{45}$, aplican un diseño mixto temporal y geográfico en el que, además de analizar por medio de regresión de Poisson la asociación entre las variaciones diarias de contaminación y de las defunciones, llevan a cabo un estudio geográfico comparando las razones estandarizadas de la mortalidad total e infantil por distritos.

Varios de los estudios aplican modelos no paramétricos como los métodos aditivos generalizados (GAM) para el examen de la forma funcional y el control de algunas variables. El primer autor, en la serie revisada, que utiliza esta aproximación es Schwartz en un estudio en el Condado de Hamilton (Cincinnati) ${ }^{46}$. La ventaja de esta aproximación sobre los modelos paramétricos es que no impone una forma de la relación «a priorim entre las variables a estudio, sino que ésta se adapta a la propia distribución de los datos. Otros autores, como Pope y Kalkstein en el Valle de Utah ${ }^{47}$, Hoeck et al en Roterdam $^{48}$, o Kelsall et al, en Filadelfia ${ }^{18}$ también han utilizado como método de elección la regresión de Poisson con modelos aditivos generalizados.

Además de los análisis complementarios que se han citado al comentar la metodolo- gía del proyecto APHEA (examen de la modificación de efecto según estación del año o semestre, construcción de modelos con varios contaminantes), son frecuentes otras profundizaciones como los análisis de sensibilidad aplicando diferentes aproximaciones metodológicas ${ }^{5,17,35,37}$ o los análisis dosis respuesta. Entre estos últimos se ha ensayado las distintas representaciones gráficas de la relación contaminante-mortalidad ${ }^{10,25,27,49}$ y la estimación de los coeficientes para distintas categorías de los niveles de contaminantes (quintiles) ${ }^{50}$.

\section{Retardos analizados}

La relación entre contaminación y mortalidad es dinámica en el tiempo y puede producirse con un cierto retraso. La ventana temporal que se analiza debe de ser limitada y guiada, en principio, por criterios de plausibilidad biológica. En el protocolo APHEA $^{44}$ se estableció por consenso explorar las asociaciones con los niveles de contaminación de hasta tres días antes (cinco para el ozono). La mayoría de trabajos se ha limitado a un retardo menor de cinco días, aunque en otros se ha llegado a analizar retardos mayores, de $9^{10}$ y hasta 20 dias $^{30}$.

Para todos los contaminantes las asociaciones más significativas se obtienen con los niveles de polución del mismo día o del día antes, siendo escasos los estudios en los que la asociación más precisa se produce con un mayor retraso.

\section{Número de captadores e imputación de valores perdidos}

Muchas de las series de contaminación analizadas presentan valores perdidos en algunos días. Un número importante de valores perdidos supone una perdida de potencia estadística, al disminuir el tamaño efectivo de la serie y, todavía peor, si se producen de forma sistemática (por ejemplo, ausencia de 
lecturas en los fines de semana) podrían llegar a ser motivo de estimaciones sesgadas.

Este es un aspecto que generalmente no es tenido en cuenta en los estudios revisados. En la mayoría de los trabajos no se da información alguna del manejo de estos valores. En algunos casos para minimizar el número de días con valores perdidos se exige que las estaciones captadoras incluidas en el análisis tengan un mínimo porcentaje de lecturas válidas (en general superior al $75 \%$ ), reemplazándose el resto con la información disponible por otras estaciones con datos válidos en el mismo día.

El número de captadores utilizado para construir las series de contaminación varía de un estudio a otro, generalmente en relación con el tamaño de la población analizada. El número de captadores se movió entre las series obtenidas con una misma estación $^{33,40,48,49}$ hasta aquellas construidas con 10 ó más monitores ${ }^{11,19,28,30}$. En algunos casos, como los estudios llevados a cabo en Filadelfia ${ }^{16-18}$, el número de estaciones variaba a lo largo de la serie.

\section{Meta-análisis}

En el primero de los cuatro meta-análisis revisados, Schwartz $z^{5}$ utiliza los datos de nueve ciudades norteamericanas y de Londres para estimar un efecto combinado de las partículas sobre el número de defunciones por todas las causas. En otro de los estudios combinados, Schwartz et $\mathrm{al}^{6}$, analizan el efecto de las partículas finas en seis ciudades del este de los Estados Unidos. En ambos casos los efectos combinados de la contaminación sobre la mortalidad se calcularon como la media de las estimaciones de las ciudades ponderadas por la inversa de la varianza. Los otros dos meta-análisis pertenecen al proyecto APHEA ${ }^{2,3}$. En este caso, los primeros cálculos se realizaban como en el caso anterior, es decir con un modelo de efectos fijos en el que los estimadores globales son una media ponderada de los coefi- cientes de regresión, siendo la ponderación inversamente proporcional a las varianzas locales. Posteriormente, se investigaba la homogeneidad de los coeficientes de cada ciudad con una prueba de ji cuadrado. Cuando la heterogeneidad era significativa se investigaba si era debida a diferentes factores constantes en el tiempo que representaran diferencias entre los niveles de salud de la población (razón estandarizada de mortalidad, porcentaje de población anciana, prevalencia del hábito tabáquico), los niveles de los contaminantes, y/o de las variables de control usando regresión lineal ponderada. Si la heterogeneidad persistía aún, se utilizaba un modelo de efectos aleatorios. Entre las ventajas de los meta-análisis APHEA respecto a otros se encuentra la ausencia de sesgos de publicación o selección, analizándose todos los resultados, y la utilización de una metodología estandarizada consensuada previamente con los grupos participantes.

\section{Estimaciones de la asociación entre contaminantes y mortalidad}

Las figuras 2, 3, 4, y 5 muestran, respectivamente, los efectos de las partículas en suspensión, el dióxido de azufre, el ozono y el dióxido de nitrógeno en los artículos revisados. Con el fin de permitir la comparabilidad de los resultados se han seleccionado para su representación únicamente los casos en los que se proporcionan los riesgos relativos de morir por todas las causas (o por todas las causas menos las externas), en toda la población (es decir, en todas las edades y ambos sexos) y para todo el periodo en estudio (y no únicamente por estaciones del año o semestres). No se han representado las estimaciones correspondientes al estudio de Polonia $^{20}$ por su escasa consistencia (RR muy inestables entre los diferentes retardos presentados).

En la figura 2 se han agrupado las estimaciones del efecto de las partículas según su tipo. Los aumentos de mortalidad asociados a un incremento de $10 \mu \mathrm{g} / \mathrm{m}^{3}$ en los niveles 
Figura 2

Estimaciones de la asociación a corto plazo entre los niveles de partículas y mortalidad por todas las causas.

Riesgos relativos (RR) e intervalos de confianza del 95\% para un incremento de $10 \mu \mathrm{g} / \mathrm{m} 3$ en los niveles medios de 24 horas para diferentes indicadores (partículas totales en suspensión, humos negros y partículas de un diámetro inferior a $10 \mu \mathrm{m}-P M 10-)$

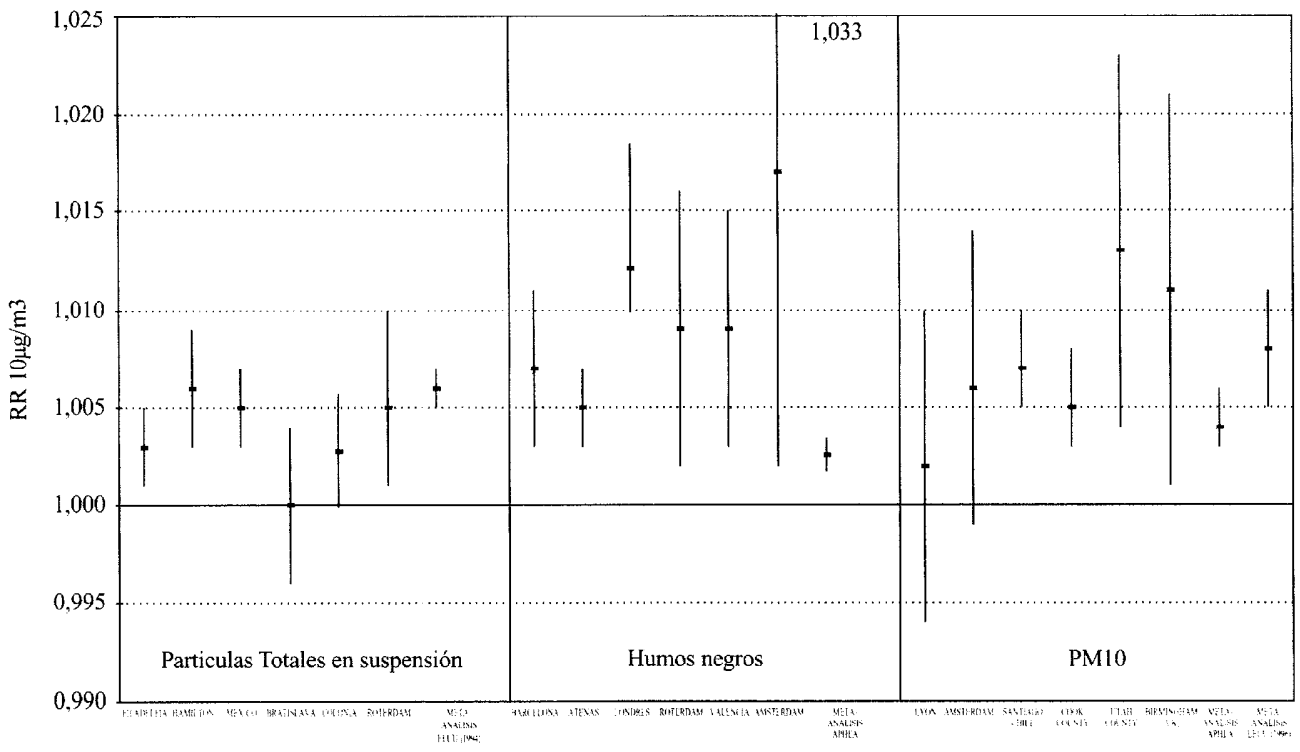

de partículas totales en suspensión (TSP) oscilan entre un incremento nulo en Bratislava ${ }^{24}$ y el $0,6 \%$ en el condado de Hamilton (Cincinnati) ${ }^{46}$. En el caso de los «humos negros» (BS) los incrementos oscilan entre un $0,5 \%$ en Atenas $^{26}$ y un $1,7 \%$ en Amsterdam ${ }^{50}$. Por último, las estimaciones encontradas para el mismo aumento de los niveles de $\mathrm{PM}_{10}$ se encuentran entre un $0,2 \%$ en $\mathrm{Lyon}^{21}$ y un $1,3 \%$ en $\mathrm{Utah}^{47}$. Todos los RR obtenidos son mayores de 1 y las asociaciones encontradas son estadísticamente significativas en 15 de los 18 casos. En la misma figura se representan los resultados de los tres meta-análisis con los resultados para partículas. En el de las nueve ciudades norteamericanas y Londres ${ }^{5}$ se obtuvo un efecto combinado de las TSP de 1,006 ( IC $_{95 \%}$ : 1,005 a 1,007), en el de las seis ciudades del este de los Estados Unidos ${ }^{6}$ el RR para PM10 fue de 1,008 (IC95\%: 1,005 a 1,011). En el meta-análisis para las partículas correspondiente al proyecto $\mathrm{APHEA}^{2}$ se obtu- vo un $\mathrm{RR}$ de morir de $1,004\left(\mathrm{IC}_{95 \%}: 1,003\right.$ a $1,006)$ para incrementos de $10 \mu \mathrm{g} / \mathrm{m} 3$ en los niveles diarios de PM10 y de $1,0026\left(\mathrm{IC}_{95 \%}\right.$ : 1,0018 a 1,0034) en los niveles de humos negros. En este último caso, el incremento fue de más del doble cuando se excluyeron del análisis las ciudades del este de Europa

Para la media diaria del dióxido de azufre se han representado los resultados de 11 estudios originales (figura 3). Se ha encontrado un RR menor a la unidad en el caso de Bratislava ${ }^{24}$ y mayor a la unidad en los otros 10 trabajos, siendo los más altos los obtenido en Barcelona ${ }^{22}$ y en Lyon ${ }^{21}$, con incrementos de mortalidad del 1,2\% en ambas ciudades asociados a aumentos en $10 \mu \mathrm{g} / \mathrm{m} 3$ de $\mathrm{SO}_{2}$. Dos de los estudios ${ }^{21.25}$ presentan estimaciones para las variaciones en los niveles de $\mathrm{SO}_{2}$ de 1 hora. En ambos casos los RR son significativamente mayores que 1 . En el meta-análisis del proyecto APHEA ${ }^{2}$ se obtuvo un RR de morir de 1,004 (IC95\%: 1,003 a 1,005) para 
Estimaciones de la asociación a corto plazo entre los niveles de dióxido de azufre $\left(\mathrm{SO}_{2}\right)$ y mortalidad por todas las causas. Riesgos relativos de mortalidad (RR) e intervalos de confianza del $95 \%$ para un incremento de $10 \mu \mathrm{g} / \mathrm{m} 3$ en los niveles medios de 24 horas de $\mathrm{SO}_{2}$

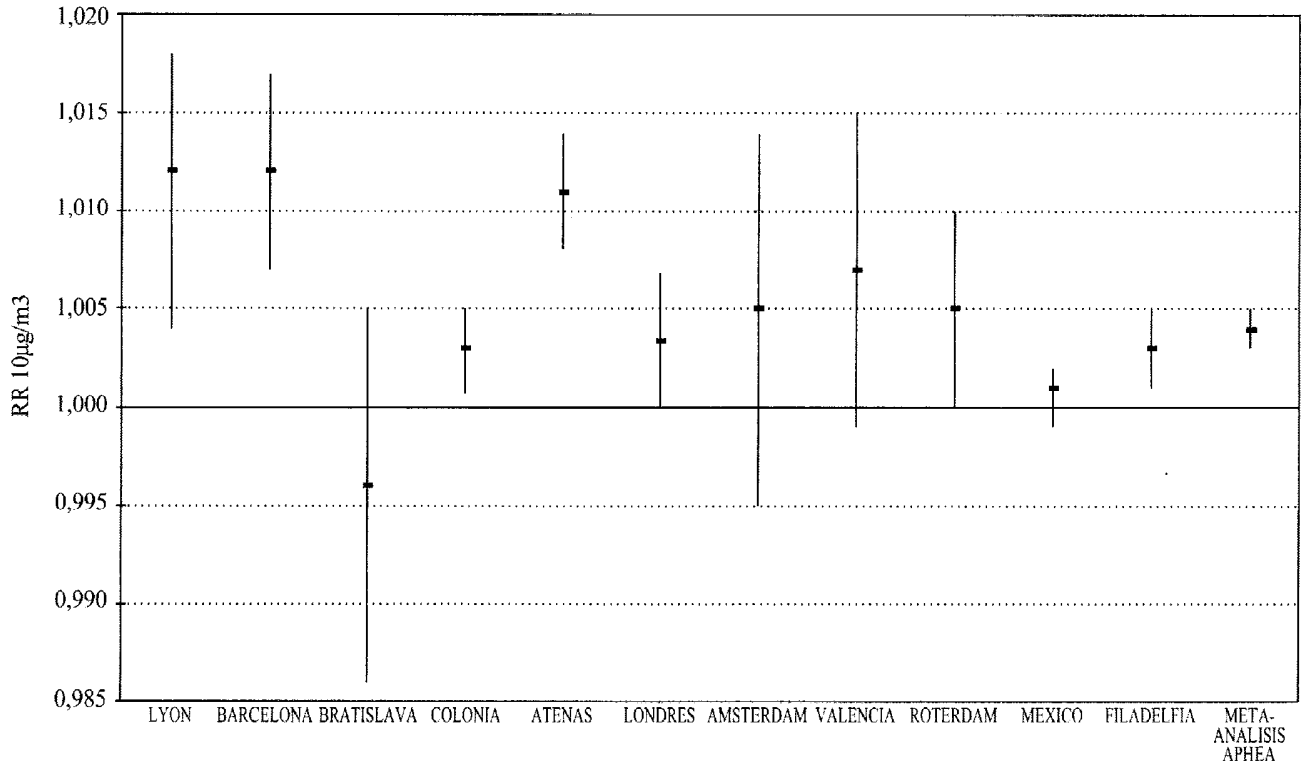

incrementos de $10 \mu \mathrm{g} / \mathrm{m} 3$ en los niveles diarios de $\mathrm{SO}_{2}$. Al igual que en el caso de las partículas, también para el SO2 los RR fueron más altos al restringir el análisis a las ciudades occidentales.

La figura 4 muestra las 10 estimaciones encontradas para tres indicadores de la contaminación por ozono. En todos los casos se han encontrado asociaciones positivas significativas excepto para Lyon ${ }^{21}$. En el meta-análisis del proyecto $\mathrm{APHEA}^{3}$ se obtuvo un RR combinado de 1,006 (IC95\%: 1,002 a 1,010 ) para el $\mathrm{O}_{3}$ de 1 hora.

De los 5 trabajos que presentan estimaciones en forma de RR acerca de la relación del dióxido de nitrógeno con la mortalidad, todas presentan valores mayores que la unidad, aunque solo en el caso de Barcelona ${ }^{22}$ se encontró una relación significativa, con un incremento de la mortalidad del $0,3 \%$ para aumentos de $10 \mu \mathrm{g} / \mathrm{m}^{3}$ en los niveles de $\mathrm{NO}_{2}$ de 1 hora (figura 5). En el meta-análisis del proyecto $\mathrm{APHEA}^{3}$ se obtuvo un RR combinado de $1,003\left(\mathrm{IC}_{95 \%}: 1,002\right.$ a 1,004$)$ para el $\mathrm{NO}_{2}$ de 1 hora.

En el caso del CO se dispone de tres estimaciones, referidas a población general, de $\mathrm{RR}$ de morir para incrementos de $1 \mathrm{mg} / \mathrm{m}^{3}$. Los incrementos asociados de mortalidad son $0,7 \%\left(\mathrm{IC}_{95 \%}, 0,3-1,1 \%\right)$ en Atenas $^{26}$, $1,1 \%\left(\mathrm{IC}_{95 \%} 0,6-1,7 \%\right)$ en Filadelfia ${ }^{18}$ y $2 \%$ $\left(\mathrm{IC}_{95 \%}-0,1-4 \%\right)$ en el estudio de Amsterdam $^{50}$.

\section{Estimaciones para causas de mortalidad específicas}

Como se comentó anteriormente en gran parte de los trabajos revisados, se analizó la asociación de la contaminación con otras causas de muerte más específicas, como la mortalidad por enfermedades respiratorias o por enfermedades circulatorias. En general el efecto encontrado para ambas causas era 


\section{Figura 4}

Estimaciones de la asociación a corto plazo entre los niveles de ozono y mortalidad por todas las causas. Riesgos relativos de mortalidad (RR) e intervalos de confianza del $95 \%$ para un incremento de $10 \mu \mathrm{g} / \mathrm{m} 3$ en los niveles medios de 24 horas,(24h) y máximos de 1 (1h) y 8 horas (8h) de ozono

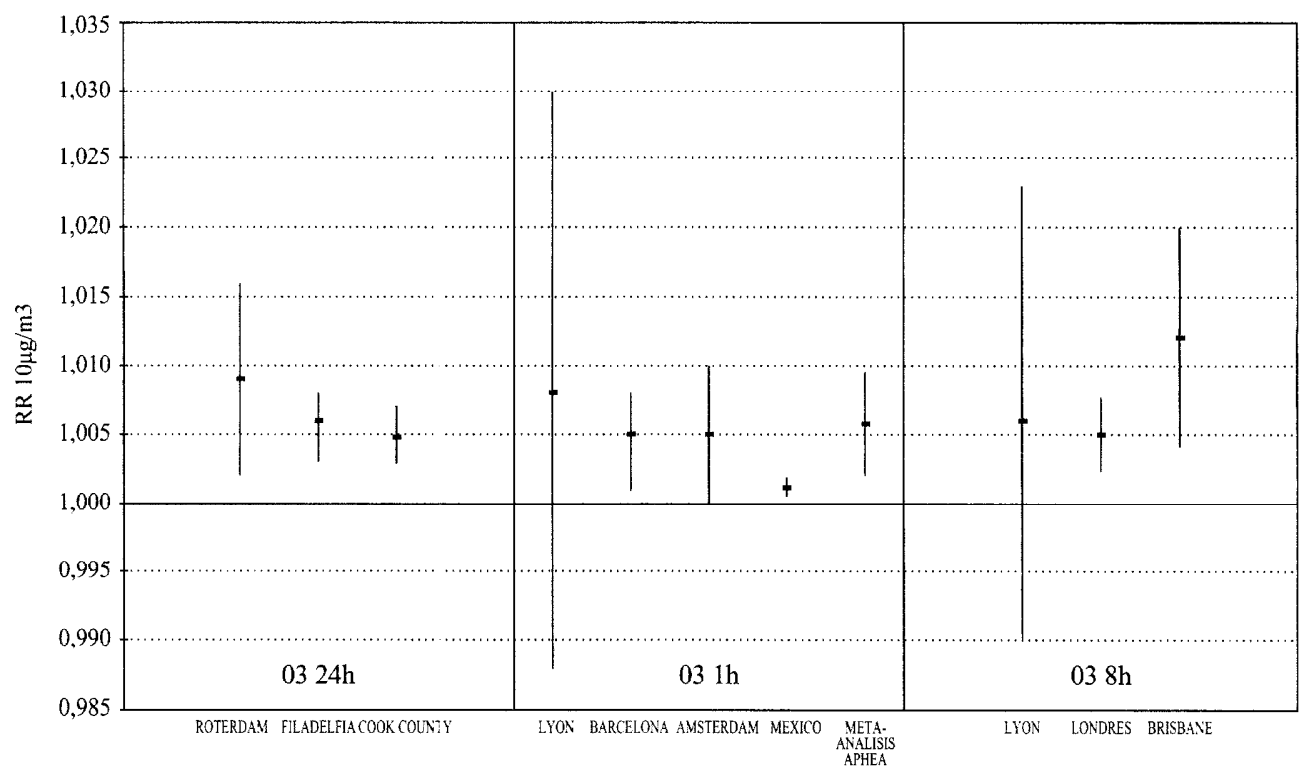

Figura 5

Estimaciones de la asociación a corto plazo entre los niveles de dióxido de nitrógeno $\left(\mathrm{NO}_{2}\right)$ y mortalidad por todas las causas. Riesgos relativos de mortalidad (RR) e intervalos de confianza del $95 \%$ para un incremento de $10 \mu \mathrm{g} / \mathrm{m} 3 \mathrm{en} \mathrm{los}$ niveles medios de 24 horas $\left(24 \mathrm{~h}\right.$ ) (izquierda) y máximos de una hora (1h) de $\mathrm{NO}_{2}$ (derecha)

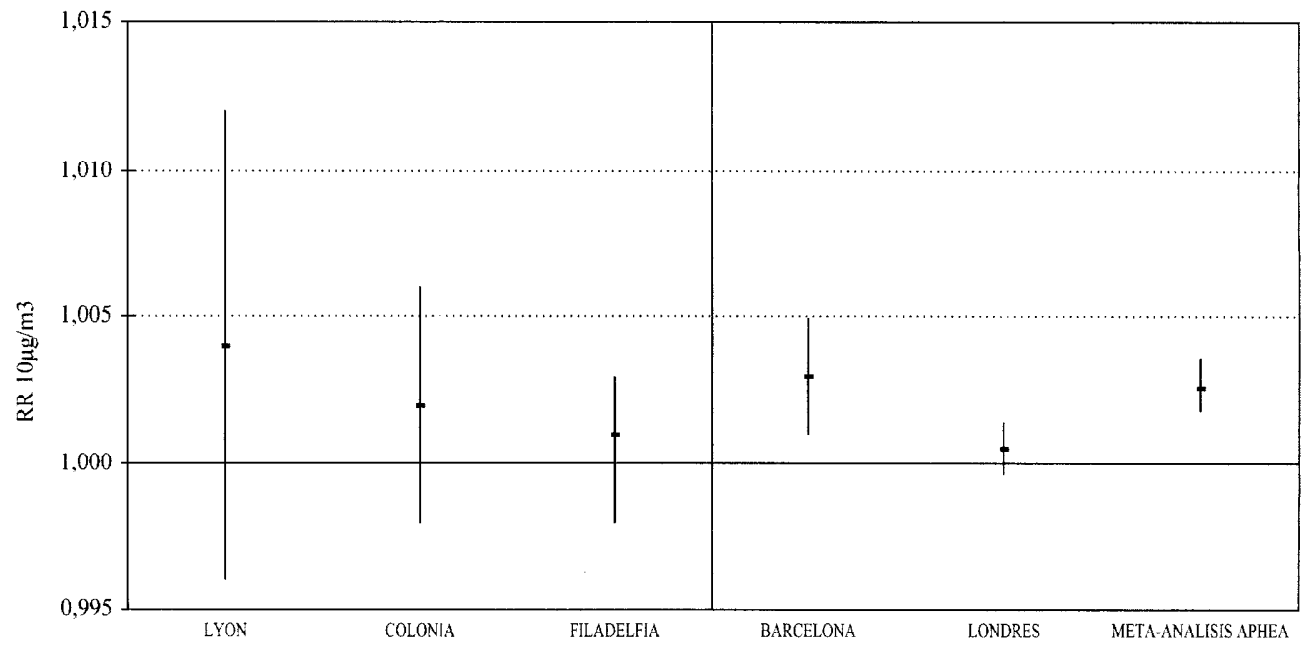


algo mayor que el obtenido para la mortalidad global $22,32,51$.

En otras ocasiones, la causa de muerte analizada era menos común. Knobel et al ${ }^{10}$ estudian el efecto de la contaminación sobre la mortalidad súbita infantil (CIE 9.798) en la isla de Taiwan. La medida de la exposición se basó en los valores diarios de la visibilidad óptica (medida correlacionada inversamente con la contaminación por partículas). El paso de la categoría con menor visibilidad a la de mayor visibilidad (de un total de cinco categorías) se asoció de forma significativa con un incremento de 5 veces de las tasas de mortalidad por esta causa.

En Sao Paulo, Pereira et al ${ }^{11}$ utilizan como indicador la mortalidad intrauterina de fetos de más de 28 semanas de gestación. El contaminante que se asoció de forma significativa con esta causa de mortalidad fue el $\mathrm{NO}_{2}$. Además, los autores encontraron una asociación positiva del monóxido de carbono en el aire con los niveles de carboxihemoglobina en las muestras de cordón umbilical.

\section{Otros diseños}

Nueve manuscritos originales que estudian la asociación entre contaminación atmosférica y mortalidad, utilizando un diseño diferente al de series temporales con el día como unidad de análisis.

Cuatro de los estudios se basan en diseños longitudinales de cohortes. Dos se llevaron a cabo en la ciudad de Roma, analizando el posible efecto de desempeñar determinadas ocupaciones como la de taxista ${ }^{52}$ o policía urbano ${ }^{53}$, en la mortalidad por determinados tipos de enfermedades. La hipótesis en la que se basan ambos trabajos es que estos colectivos están más expuestos a la contaminación atmosférica, debido a su profesión, que el resto de ciudadanos. Se detectó un exceso de mortalidad para algunos tipos de cáncer, aunque el hecho de no valo- rar directamente la exposición limita en parte las conclusiones.

Wodruff et $\mathrm{al}^{9}$ exploran la posible relación entre la exposición a la contaminación atmosférica por partículas finas en los dos primeros meses de vida con la mortalidad infantil en una cohorte de 4 millones de nacidos en Estados Unidos. Un incremento en los niveles de partículas de $10 \mu \mathrm{g} / \mathrm{m} 3$ se asoció de forma significativa con un incremento del $4 \%\left(\mathrm{IC}_{95 \%} 2-7 \%\right)$ en la mortalidad por todas las causas y del $12 \% \quad\left(\mathrm{IC}_{95 \%} 7-17 \%\right)$ en la debida a muerte súbita infantil. También se encontró un efecto similar sobre la mortalidad respiratoria, aunque solo en el grupo de niños con peso normal al nacimiento. Cuando el indicador de mortalidad eran otras causas diferentes a la muerte súbita infantil $y$ por enfermedades respiratorias el efecto era nulo $(R R=1)$.

El cuarto estudio de cohortes se llevó a cabo también en $\mathrm{EEUU}^{8}$ y consistió en el seguimiento de más de medio millón de adultos (mayores de 30 años) procedentes de 151 áreas metropolitanas. A cada individuo se le asignó el nivel de contaminación de su ciudad con un retardo aproximado de 9 años y se determinó su estado vital. Los niveles de sulfatos y partículas finas se asociaron significativamente con la mortalidad por todas las causas, de manera que un incremento en $10 \mu \mathrm{g} / \mathrm{m} 3$ se asoció con un aumento paralelo en el riesgo de morir del 7\% para ambos contaminantes. Para la mortalidad por enfermedades circulatorias la asociación fue de mayor magnitud, con incrementos del $12,3 \%\left(\mathrm{IC}_{95 \%} 7,7\right.$ a $\left.17,1 \%\right)$ y del $11,7 \%$ $\left(\mathrm{IC}_{95 \%} 6,6\right.$ a $\left.16,7 \%\right)$ para aumentos en 10 $\mu \mathrm{g} / \mathrm{m} 3$ de los niveles de sulfatos y partículas finas respectivamente.

Una de las grandes ventajas de estos estudios es la capacidad de poder ajustar los riesgos por determinados factores individuales, como la edad, el sexo, tabaquismo, masa corporal, consumo de alcohol, etcétera. A pesar de que la medida de la exposición sigue haciéndose por asignación indivi- 
dual de una variable originalmente ecológica, el efecto del posible error de mala clasificación se comporta de igual manera que en las mediciones genuinamente individuales ${ }^{54}$.

Tres trabajos consisten en estudios ecológicos geográficos, con el denominador común de analizar la relación entre contaminación atmosférica y mortalidad por cáncer de pulmón. Los dos realizados en Japón ${ }^{55,56}$ encuentran una asociación estadísticamente significativa de los niveles de $\mathrm{NO}_{2}$ con la mortalidad por esta causa. Una de las limitaciones, aparte de las propias de este tipo de diseños, es el hecho de haber analizado solamente dos contaminantes $\left(\mathrm{SO}_{2}\right.$ y $\left.\mathrm{NO}_{2}\right)$, por lo que no puede descartarse el efecto de otros compuestos. El tercer estudio geográfico se llevó a cabo en Escocia ${ }^{57}$, comparando la mortalidad en 12 localidades expuestas en relación a otras tantas no expuestas. La denominación de expuesta o no expuesta se basó en criterios indirectos según las fuentes locales de contaminación y no en la medición directa de la misma. Se halló un efecto significativo sobre la mortalidad global aunque no sobre la debida a cáncer de pulmón.

Ames et $\mathrm{al}^{58}$ analizan la relación entre mortalidad y la utilización de defoliantes de algodón en el Valle de San Joaquín (California). La variable dependiente fue la proporción de mortalidad respiratoria ocurrida en el trimestre de septiembre a noviembre respecto al resto del año, la cual se comparó entre las zonas según utilizaran o no defoliantes de algodón. También analizaron la relación con la contaminación atmosférica y hallaron un efecto significativo de las partículas y óxidos de nitrógeno (NOx). Aunque el periodo de estudio era bastante amplio (1970-1990) se trata de una serie con pocas observaciones (total 21) ya que la unidad de análisis era el trimestre. Al no incluir en el análisis otras posibles variables de confusión como las meteorológicas no podemos descartar su implicación en los hallazgos obtenidos.
Por último, tenemos un trabajo que estudia el impacto en la mortalidad de un cpisodio ocurrido en Londres en la semana del 12 al 18 de diciembre de $1991^{59}$. Esta semana se caracterizó por un incremento abrupto de los niveles de $\mathrm{NO}_{2}$, alcanzando sus valores horarios cifras superiores a los $800 \mu \mathrm{g} / \mathrm{m} 3$. La semana del episodio fue comparada con la anterior y con las mismas dos semanas en los 4 años previos. También analizaron tres áreas geográficas de control, para descartar que el fenómeno no se hubiera producido solamente a nivel local. El principal resultado fue que hubo un incremento significativo en la mortalidad global esperada en esa semana del $10 \%$. El episodio fue introducido en el modelo como una variable indicadora, por lo que no se puede descartar el efecto de otros fenómenos coincidentes (hubo un descenso marcado de la temperatura y un aumento de los niveles de humos negros, aunque no tan marcado como el de $\mathrm{NO}_{2}$ ).

\section{DISCUSIÓN}

En la gran mayoría de estudios revisados predominan las asociaciones positivas entre contaminación atmosférica y mortalidad. Dicho de otro modo, estamos ante una asociación que es consistente, al reproducirse en diferentes entornos y con diferentes metodologías.

Quizá el termino de «hallazgo consistente» sea aplicable, sobre todo, a las partículas, ya que son el contaminante más analizado. Tanto los estudios por ciudades como los meta-análisis muestran un efecto de las partículas sobre la mortalidad por todas las causas que no solamente es positivo y significativo sino que se sitúa en una banda relativamente estrecha. A pesar de ello, cuando se analiza un indicador como es el $\mathrm{PM}_{10}$ se encuentran efectos ligeramente superiores en las ciudades americanas ${ }^{5,6}$ que en las europeas ${ }^{2}$. Al comparar los resultados obtenidos entre los distintos indicadores de partículas parece observarse que la asociación entre los indicadores de partículas más finas $\left(\mathrm{PM}_{10} \mathrm{y}\right.$ humos 
negros) es algo mayor que la obtenida para las partículas totales en suspensión. Sin embargo, los pocos estudios que han utilizado medidas de partículas más finas ${ }^{37.45}$ no apoyan dicha hipótesis. En los estudios de cohortes $^{8,9}$ se obtiene una asociación entre partículas y mortalidad varias veces superior a los de series temporales, ello podría ser interpretado como una prueba de la existencia de efectos crónicos que se suman al impacto a corto plazo de la contaminación. Sin embargo, simplemente con las evidencias actuales es problemático realizar inferencias sobre respuestas a largo plazo.

Casi toda la información encontrada sobre los efectos a corto plazo del $\mathrm{SO}_{2}$ en la mortalidad, procede de estudios europeos. Aparte de éstos, únicamente se dispone del resultado de uno de los trabajos sobre las series de Filadelfia ${ }^{18}$ y del realizado en Méxi$\mathrm{co}^{38}$. Aunque tradicionalmente este contaminante ha recibido poca atención en Norteamérica, en el trabajo de Filadelfia citado los resultados obtenidos son similares a los de las ciudades europeas.

E1 resto de contaminantes se han analizado con menor frecuencia que las partículas, aunque también muestran un efecto significativo sobre la mortalidad. Aunque el menor número de trabajos recomienda prevención antes de aventurar hipótesis, los resultados para el $\mathrm{NO}_{2}$ sugieren un efecto más claro para el indicador de 1 hora que para el acumulado de 24 horas. Para el ozono, únicamente los resultados de una ciudad, Lyon $^{21}$, han dado asociaciones no significativas. En algunos casos los autores no proporcionan las estimaciones obtenidas con estos contaminantes.

Una consideración general a la hora de hacer una valoración conjunta de estos trabajos debería ser el sesgo derivado de la no presentación de los resultados no significativos por parte de algunos autores. Sin embargo, aún valorando este posible sesgo de publicación parece muy improbable que el conjunto de resultados se deba exclusivamente al azar.
Por otro lado se ha de tener en cuenta que los resultados presentados son, por lo general, los más significativos dentro de los diferentes retardos explorados. Esta situación puede enmascarar la estructura real de la relación estudiada, que podría ser de importancia a la hora de plantear estrategias efectivas para reducir los efectos de la contaminación ${ }^{60}$.

Desde el punto de vista metodológico predomina la utilización de modelos de regresión log-lineales de Poisson. En algunos estudios sc incorporan al modclo términos no paramétricos no lineales, que permiten una mayor flexibilidad en la modelización de variables que, como la temperatura y la humedad, pueden adoptar diferentes formas funcionales. Estos modelos aditivos generalizados (GAM), son utilizados en la actualidad con una frecuencia cada vez mayor y es previsible que en un futuro próximo sean el estándar de análisis para este tipo de estudios.

Entre las limitaciones, destacaríamos, por un lado, que estos estudios son en su gran mayoría ecológicos por lo que debemos ser precavidos a la hora de extrapolar los hallazgos a un nivel individual. Sin embargo, se han podido confirmar mediante diseños individuales los hallazgos obtenidos en los estudios ecológicos ${ }^{7-9}$. Por otro lado, en prácticamente todos los trabajos revisados la exposición se ha medido a partir de los datos obtenidos en monitores fijos. Los niveles calculados de esta manera no necesariamente han de ser representativos de la exposición de las poblaciones y/o de los individuos estudiados. Sin embargo, cuando el estudio es de series temporales, no es tan importante la representatividad de la exposición si no que las variaciones en los niveles medidos se correlacionen con los reales ${ }^{61}$.

Desde el punto de vista del control de la posible confusión o modificación de efecto por parte de otros factores los dos puntos cruciales son el efecto de las variaciones estacionales y el control de los factores meteorológicos, especialmente de la temperatura 
y de su interacción con los contaminantes. Los estudios realizados en Filadelfia ${ }^{16-18,62}$ demuestran que utilizar diferentes métodos para controlar estos factores resulta en coeficientes distintos.

Otro asunto importante a considerar es la correlación entre contaminantes, con los problemas de multicolinealidad que esto conlleva. No se debe olvidar que las estimaciones que se han expuesto en la tabla 3 son las obtenidas con los modelos con un solo contaminante. Estos resultados deberían ser vistos como «índices» de la contaminación conjunta, sin indicar necesariamente que el contaminante con la mayor significación sería el mejor candidato sobre el que establecer medidas de control. En todo caso, se deberían explorar modelos con más de un contaminante. Algunos autores recomiendan probar combinaciones de dos contaminantes o realizar análisis estratificado de cada uno de ellos según los niveles de los otros ${ }^{60}$.

Uno de los aspectos que aún no ha recibido mucha atención es el análisis de la relación dosis/respuesta entre los niveles de contaminación y el riesgo estimado. La posibilidad de encontrar un umbral por debajo del cual no se suponga un efecto significativo de la contaminación tiene implicaciones importantes sobre todo a la hora de establecer límites de seguridad. A pesar de que los estudios ecológicos presentan dificultades para detectar valores umbrales ${ }^{63}$ es importante, al menos, comprobar o descartar la linealidad de las relaciones, bien de forma gráfica o mediante la aplicación de alguna prueba estadística ${ }^{47}$.

En definitiva, a pesar de las limitaciones expuestas, se ha de tener en cuenta que en la mayoría de trabajos revisados se han aplicado métodos adecuados y con una perspectiva amplia, con un buen ajuste estacional y control de los efectos retardados de los contaminantes y las variables meteorológicas y, por último, se han investigado métodos alternativos para comprobar la robustez de las estimaciones. Todo lo anterior puede ayudar a concluir que existe una asociación a corto plazo entre los incrementos actuales en los niveles de contaminantes atmosféricos urbanos y el número de defunciones diarias. Ello no nos debería hacer olvidar que la asociación estadística no debe considerarse aisladamente como demostración de causalidad, sino que se necesitan otros argumentos como las evidencias de estudios toxicológicos y la plausibilidad de las respuestas fisiopatológicas.

\section{AGRADECIMIENTOS}

A Manuel Arranz y Miguel Feliu (documentalistas del IVESP).

\section{BIBLIOGRAFIA}

1. Working Group on Public Health and Fossil-Fuel Combustion. Short-term improvements in public health on fossil-fuel combustion: an interim report. Lancet 1997;350:1341-9.

2. Katsouyanni K, Touloumi G, Spix C, Schwartz J, Balducci F, Medina S, et al. Short-term effects of ambient sulphur dioxide and particulate matter on mortality in 12 European cities: results from time series data from the APHEA project. Air Pollution and Health: a European Approach. BMJ 1997;314:1658-63.

3. Touloumi G, Katsouyanni K, Zmirou D, Schwartz J, Spix C, Ponce de Leon A, Tobias A, Quénel P, Rabczenko D, Bacharova L, et al. Short-term Effects of Ambient Oxidant Exposure on Mortality: A Combined Analysis within the APHFA Project Am J Epidemiol. 1997;146:177-85.

4. Zmirou D, Schwartz J, Saez M, Zanobetti A, Wojtyniak B, Touloumi G, Spix C. Ponce de Leon A, et al. Time-series analysis of air pollution and cause-specific mortality. Fpidemiol 1998;9:495-503

5. Schwartz J. Air pollution and daily mortality: a review and meta analysis. Environ Res 1994; $64: 36-52$.

6. Schwartz J, Dockery D, Neas L. Is daily mortality associated specifically with fine particles? J Air Waste Manage Assoc 1996;46:927-39.

7. Dockery D, Pope C, Xu X, Spengler J, Ware J, Fay M, et al. An association between air pollution and mortality in six U.S. cities. N Engl $\mathrm{J}$ Med 1993;329:1753-9. 
8. Pope C, Thun M, Namboodiri M, Dockery D, Evans J, Speizer F, et al. Particulate air pollution as a predictor of mortality in a prospcctive study of U.S. adults. Am J Respir Crit Care Med 1995;151:669-74.

9. Woodruff T, Grillo J, Schoendorf K. The relationship between selected causes of postneonatal infant mortality and particulate air pollution in the United States. Environ Health Perspect 1997; 105:608-12.

10. Knobel H, Chen C, Liang K. Sudden infant death syndrome in relation to weather and optimetrically measured air pollution in Taiwan. Pediatrics 1995;96:1106-10.

11. Pereira L, Loomis D, Conceiao G, Braga A, Arcas R, Kiski H, Böhm G, Saldiva P. Association between air pollution and intrauterine mortality in Sao Paulo, Brazil. Environ Health Perspect 1998; 106:325-9.

12. Ballester F, Merino C, Pérez Hoyos S. La asociación entre contaminación atmosférica y mortalidad: una revisión de los estudios epidemiológicos recientes. Rev Esp Salud Pública 1995;69:177-88.

13. Editorial. La contaminació atmosfèrica i la salut. Gaseta Sanitària de Barcelona 1984; Sèrie Monografies $2: 3-5$.

14. Díaz Jiménez J, Alberdi Odriozola J, Montero Rubio J, Mirón Pérez I. Asociación entre la conlaminación atmosférica por dióxido de azufre y partículas totales en suspensión y la mortalidad en la ciudad de Madrid (1986-1992). Gac Sanit 1998;12:203-11.

15. Tobias A, Sunyer J, Castellsague J, Sáez M, Antó J. Impacto de la contaminación atmosférica sobre la mortalidad y las urgencias por enfermedad pulmonar obstructiva crónica y asma en Barcelona. Gac Sanit 1998;12:219-26.

16. Schwartz J. What are People Dying of on High Air Pollution Days? Environ Res 1994;64:26-35.

17. Moolgavkar S, Luebeck E, Hall T, Anderson E. Air pollution and daily mortality in Philadelphia. Epidemiol 1995;6:476-84.

18. Kelsall J, Samet J, Zeger S, Xu J. Air pollution and mortality in Philadelphia, 1974-1988. Am J Epidemiol 1997;146:750-62.

19. Dab W, Medina S, Quénel P, Le Moullec Y, Le Tertre A, Thelot B, et al. Short term respiratory health effects of ambient air pollution: results of the APHEA project in Paris. J Epidemiol Community Health 1996;50 (Suppl 1):s42-6.
20. Wojtyniak B, Piekarski T. Short term effect of air pollution on mortality in Polish urban populations - what is different? J Epidemiol Community Health 1996;50 (Suppl 1):s36-41.

21. Zmirou D, Barumandzadeh T, Balducci F, Ritter P, Laham G, Ghilardi J. Short term effects of air pollution on mortality in the city of Lyon, France, 1985-90. J Epidemiol Community Health 1996; 50 (Suppl 1):s30-5.

22. Sunyer J, Castellsague J, Sáez M, Tobias A, Antó J. Air pollution and mortality in Barcelona. J Epidemiol Community Health 1996:50 (Suppl 1):s76-s80

23. Vigotti M, Rossi G, Bisanti L, Zanobetti A, Schwartz J. Short term effects of urban air pollution on respiratory health in Milan, Italy, 1980-89. J Epidemiol Community Health 1996; 50 (Suppl 1):s71-5.

24. Bacharova L, Fandakova K, Bratinka J, Budinska M, Bachar J, Gudába M. The association between air pollution and the daily number of deaths: findings from the Slovak Republic contribution to the APHEA project. J Epidemiol Community Health 1996;50 (Suppl 1):s19-21.

25. Spix C, Wichmann H. Daily mortality and air pollutants: findings from Köln, Germany. J Epidemiol Community Health 1996;50 (Suppl 1): s52-8.

26. Touloumi G, Samoli E, Katsouyanni K. Daily mortality and "winter type" air pollution in Athens, Greece - a time series analysis within the APHEA project. J Epidemiol Community Health 1996;50 (Suppl 1):s47-51.

27. Anderson H, Ponce de Leon A, Bland J, Bower J, Strachan D. Air pollution and daily mortality in London: 1987-92. BMJ 1996;312:665-9.

28. Sartor F, Snacken R, Demuth C, Walckiers D. Temperature, ambient ozone levels, and mortality during summer 1994, in Belgium. Environ Res 1995;70:105-13.

29. Sartor F, Demuth C, Snacken R, Walckiers D. Mortality in the elderly and ambient ozone concentration during the hot summer, 1994, in Belgium. Environ Res 1997;72:109-17.

30. Saldiva P, Lichtenfels A, Paiva P, Barone I, Martins M, Massad E, et al. Association between air pollution and mortality due to respiratory diseases in children in Sao Paulo, Brazil: a preliminary report. Environ Res 1994;65:218-25.

31. Organización Mundial de la Salud. OPS, editor. Manual de la Clasificación Estadística Internacional de Enfermedades, Traumatismos y Causas de Defunción. 9. a revisión. Publicación núm 353. Washington: Organización Mundial de la Salud; 1978. 
32. Xu X, Gao J, Dockery D, Chen Y. Air pollution and daily mortality in residential areas of Beijing, China. Arch Environ Health 1994:49:216-22.

33. Styer P, McMillan N, Gao F, Davis J, Sacks J. Effect of outdoor airborne particulate matter on daily death counts. Fnviron Health Perspect 1995;103:490-7.

34. Ito K, Thurston G. Daily PM10/Mortality associations: an investigation of at-risk subpopulations. J Expo Anal Env Epid 1996;6:79-95.

35. Saldiva P, Pope C, Schwartz J, Dockery D, Lichtenfels A, Salge J, ct al. Air pollution and mortality in elderly people: a time-series study in Sao Paulo, Brazil. Arch Environ Ilealth 1995;50:159-63.

36. Ostro B, Sánchez J, Aranda C, Eskeland G. Air pollution and mortality: results from a study of Santiago, Chile. J Expo Anal Env Epid 1996;6:97-114.

37. Ostro B. Fine particulate air pollution and mortality in two Southern California counties. Environ Res 1995;70:98-104.

38. Borja-Aburto V, Loomis D, Bangdiwala S, Shy C, Rascon Pacheco R. Ozone, suspended particulates, and daily mortality in México City. Am J Epidemiol 1997;145:258-68.

39. Peters A, Wichmann H, Tuch T, Heinrich J, Heyder J. Respiratory Effects Are Associated with the Number of Ultrafine Particles. Am J Respir Crit Care Med 1997;155:1376-83.

40. Rahlenbeck S, Kahl H. Air population and mortality in East Berlin during the winters of 1981-1989. Int J Epidemiol 1996;25:1220-6.

41. Ballester F, Corella D, Pérez-Hoyos S, Hervás A. Air pollution and mortality in Valencia, Spain: a study using the APHEA methodology. J Epidemiol Community Health 1996;50:527-33.

42. Simpson R, Williams G, Petroeschevsky A, Morgan G, Rutherford S. Associations between outdoor air pollution and daily mortality in Brisbane, Australia. Arch Environ Health 1997;52:442-54.

43. Schwartz J, Spix C, Touloumi G, Bacharova L, Barumandzadeh T, Le Tertre A, et al. Methodological issucs in studies of air pollution and daily counts of deaths or hospital admissions. J Epidemiol Commun I Iealth 1996;50 (Suppl 1):S3-S11

44. Katsouyanni K, Schwartz J, Spix C, Touloumi G, Zmirou D, Zanobetti A, et al. Short term effects of air pollution on health: a European approach using epidemiologic time series data: the APHEA protocol. J Epidemiol Community Health 1996; 50 (Suppl 1):s12-8.
45. Salinas M, Vega J. The effect of outdoor air pollution on mortality risk: an ecological study from Santiago, Chile. World Health Stat Q 1995;48: $118-25$.

46. Schwartz J. Total suspended particulate matter and daily mortality in Cincinnati, Ohio. Environ Health Perspect 1994;102:186-9.

47. Pope C, Kalkstein L. Synoptic weather modeling and estimates of the exposure-response relationship between daily mortality and particulate air pollution. Environ Health Perspect 1996;104:414-20.

48. Hock G, Schwartz J, Groot B, Eilers P. Effects of ambient particulate matter and ozone on daily mortality in Rotterdam, The Netherlands. Arch Environ Health 1997;52:455-63.

49. Wordley J, Walters S, Ayres J. Short term variations in hospital admissions and mortality and particulate air pollution. Occup Environ Med 1997;54:108-16.

50. Verhoeff A, Hoek G, Schwartz J, Van Wijnen J. Air pollution and daily mortality in Amsterdam. Epidemiol 1996;7:225-30.

51. Touloumi G, Pocock S, Katsouyanni K, Trichopoulos D. Short-term effects of air pollution on daily mortality in Athens: a time-series analysis. Int J Epidemiol 1994;23:957-67.

52. Borgia P, Forastiere F, Rapiti E, Rizzelli R, Magliola M, Perucci C, Axelson O. Mortality among taxi drivers in Rome: a cohort study. Am J Ind Med 1994;25:507-17.

53. Forastiere F, Perucci C, Di Pietro A, Miceli M, Rapiti E, Bargagli A, Borgia P. Mortality among urban policemen in Rome. Am J Ind Med $1994 ; 26: 785-98$.

54. Künzli N, Tager I. The Semi-individual study in air pollution epidemiology: a valid design as compared to ecologic studies. Environ Health Perspect 1997;105:1078-83.

55. Tango T. Effect of air pollution on lung cancer: a Poisson regression model based on vital statistics. Environ Health Perspect 1994;102 Suppl 8:s41-5.

56. Choi K, Inoue S, Shinozaki R. Air pollution, temperature, and regional differences in lung cancer mortality in Japan. Arch Environ Health $1997 ; 52: 160-8$

57. Williams F, Ogston S, Lloyd O. Sex ratios of births, mortality, and air pollution: can measuring the sex ratios of births help to identify health hazards from air pollution in industrial environments? Occup Environ Med 1995;52: 164-9. 
58. $\Lambda$ mes R, Gregson J. Mortality following cotton defoliation: San Joaquin Valley, California, 1970- 1990. J Occup Environ Med 1995;37: 812-9.

59. Anderson H, Limb E, Bland J, Ponce de Leon A, Strachan D, Bower J. Health effects of an air pollution episode in London, December 1991. Tho$\operatorname{rax} 1995 ; 50: 1188-93$.

60. Lipfert F, Wyzga R. Uncertainties in Identifying responsible pollutants in observational epidemiology studies. Inhal Toxicol 1995;7:671-89.
61. Janssen N, Hoek G, Brunekreef B, Harssema H, Mensink I, Zuidhof A. Personal sampling of particles in adults: relation among personal, indoor, and outdoor air concentrations. Am J Epidemiol 1998; 147:537-47.

62. Moolgavkar S, Luebeck E. A critical review of the evidence on particulate air pollution and mortality. Epidemiology 1996;7:420-8.

63. Watt M, Godden D, Cherrie J, Seaton A. Individual exposure to particulate air pollution and its relevance to tresholds for health effects: a study of traffic wardens. Occup Environ Med 1995; 52: 790-2. 